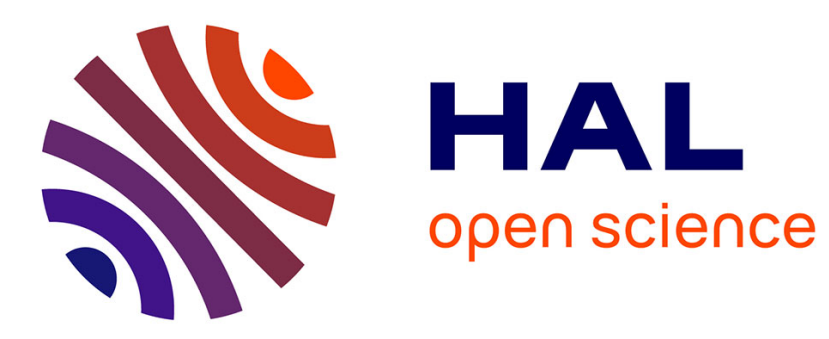

\title{
A data fusion environment for multimodal and multi-informational neuronavigation
}

Pierre Jannin, Olivier J. Fleig, E. Seigneuret, Christophe Grova, Xavier

Morandi, Jean-Marie Scarabin

\section{- To cite this version:}

Pierre Jannin, Olivier J. Fleig, E. Seigneuret, Christophe Grova, Xavier Morandi, et al.. A data fusion environment for multimodal and multi-informational neuronavigation. Computer Aided Surgery, 2000, 5 (1), pp.1-10. 10.3109/10929080009148866 . inserm-00331756

\section{HAL Id: inserm-00331756 https://www.hal.inserm.fr/inserm-00331756}

Submitted on 17 Oct 2008

HAL is a multi-disciplinary open access archive for the deposit and dissemination of scientific research documents, whether they are published or not. The documents may come from teaching and research institutions in France or abroad, or from public or private research centers.
L'archive ouverte pluridisciplinaire HAL, est destinée au dépôt et à la diffusion de documents scientifiques de niveau recherche, publiés ou non, émanant des établissements d'enseignement et de recherche français ou étrangers, des laboratoires publics ou privés. 


\title{
A Data Fusion Environment for Multimodal and Multi-Informational Neuro- Navigation
}

\author{
P. Jannin", Ph. D., O. J. Fleig, M.Sc., E. Seigneuret, M.D., C. Grova, M.Sc., X. Morandi, M.D., and J.M. \\ Scarabin M.D. \\ Laboratoire SIM, UPRES E.A. 2232, Université de Rennes (P.J., O.J.F., E.S., C.G., X.M., J.M.S.) and \\ Neurosurgery Department, University Hospital Rennes France (E.S., X.M., J.M.S.) and Carl Zeiss, 60, Route de \\ Sartrouville, 78230 Le Pecq France (O.J.F.)
}

\begin{abstract}
Objective: A part of planning and performing neurosurgery consists in determining target areas, areas to be avoided, landmark areas and trajectories, all components of the surgical script. Nowadays neurosurgeons have access to multimodal medical imaging to support the definition of the surgical script. The purpose of this paper is to present a software environment we have developed that allows full multimodal and multi-informational planning as well as neuro-navigation for epilepsy and tumoral surgery.

Materials and Methods: We have developed a data fusion environment dedicated to neuronavigation around the Surgical Microscope Neuronavigator system (Carl Zeiss, Oberkochen/Germany). This environment includes registration, segmentation, 3D visualization and interaction-applied tools. It provides the neuronavigation system with the multimodal information involved in the definition of the surgical script: lesional areas, sulci, ventricles segmented from magnetic resonance imaging (MRI), vessels segmented from magnetic resonance angiography (MRA), functional areas from magneto-encephalography (MEG) and functional magnetic resonance imaging (fMRI) for somato-sensory, motor or language activation. This data is considered to be relevant for the performance of the surgical act. The definition of each results from the same procedure: registration with the anatomical MRI data set defined as the reference data set, segmentation, fused 3D display, selection of the relevant entities for the surgical step, encoding in 3D surface-based representation and storage of the 3D surfaces into a file recognized by the neuro-navigation software (STP 3.4, Leibinger; Freiburg/Germany).

Results: Multimodal neuronavigation is illustrated with two clinical cases for which multimodal information was injected into the neuronavigation system. Lesional areas were used to define and follow the surgical path. Sulci and vessels helped identify the anatomical environment of the surgical field. And finally MEG and fMRI functional information helped determine the position of functional high risk areas.

Conclusion: In this short experience, being able to access preoperative multi-functional and anatomical data within the neuronavigation system was a valuable support for the surgical act.
\end{abstract}

Keywords: Multimodal Neuronavigation, Data Fusion, Pre-operative Planning, Image-Guided Neurosurgery

\section{Introduction}

In neurosurgery, an increasing number of surgical acts is defined on the basis of multimodal 3D imaging. During the initial planning phase, the neurosurgeon defines target areas, functional and anatomical high risk areas to be avoided, reference areas and trajectories to reach his target. These areas or entities are part of the surgical script, which covers every step from skin incision to the actual therapeutic procedure. In this context 3D imaging offers a spatial representation of anatomy that lets you define the location of these entities very accurately. Registration tools establish correspondence between coordinate systems of different imaging modalities. Thanks to registration, one can take advantage of the complementary nature of imaging modalities. Three-dimensional multimodal information all comes from preoperative imaging. This information, combined with atlases and the knowledge of the surgeon, leads to the definition of the surgical script. In this way, the contours of a lesion, a sulcus segmented from MRI, a blood vessel from MRA, or equivalent current dipoles from MagnetoEncephalography might determine landmarks for the surgical script. To execute the prepared scenario, the neurosurgeon needs to retrieve these entities within his surgical environment. Three dimensional neuronavigation systems establish correspondence between patient and world coordinate systems and therefore register the patient in the operating theater with preoperative multimodal imaging. We have defined multimodal and multi-informational neuronavigation as the retrieval of information (entities or objects) coming from a preoperative multimodal imaging in a neuro-navigation system. "Multi-informational" describes the fact that

Corresponding author: Pierre JANNIN, Laboratoire SIM, UPRES E.A. 2232, Faculté de Médecine, Université de Rennes I, 2, Avenue du Pr. Léon Bernard 35043 Rennes cedex France. E-Mail: pierre.jannin@univ-rennes1.fr; http://sim3.univ-rennes1.fr 
different kinds of information can be extracted from one single modality, e.g. from anatomical MRI, one can segment lesions for target areas, vessels for areas to avoid, ventricles, or sulci for reference areas.

Many papers have previously been published on the use of functional images within neuro-navigation systems $4,5,6,12,14,15$. In ${ }^{15}$, Roberts et al. outline two benefits from this integration: 1 ) no need to perform invasive intraoperative electrical mapping procedures, 2) risky open surgery might be replaced by biopsy evaluation prior to radiotherapy and/or chemotherapy. In ${ }^{14}$, Rezaï et al. describe the use of MEG information in their stereotactic image-guided neurosurgery system. Several advantages have been reported: smaller craniotomy and safer trajectory by decreasing the risk of functional morbidity. In ${ }^{12}$, Maldjian et al. emphasize the fact that functional information in a neuronavigation system identifies critical structures that are not visible via the usual surgical exposure. In ${ }^{4,5}$, Ganslandt et al. demonstrate that for a set of 50 patients the integration of MEG information into neuronavigation systems improves the functional outcome for surgery around eloquent brain areas.

The objective of this paper is to present a software environment we have developed for full multimodal and multi-informational neuronavigation. We extracted the relevant entities (for the neurosurgeon) from the different modalities available (anatomical and functional, images and signals), automating the segmentation and registration steps as much as possible. Particular attention was paid to define an "open solution". By "open" we mean extendible towards new modalities, information or entities coming from previous modalities, as well as using standard formats and being independent from the neuronavigation system (3D localizer, workstation, ...). This latter point is ensured by separating the planning functionality from navigation. We present how we have integrated this environment into the SMN neuro-navigation system from Carl Zeiss (Oberkochen/Germany). We also show some first clinical results and analyze the interest and limits of our approach.

\section{Materials and Methods}

In this section we describe our approach to multimodal neuronavigation. Information required for the planning of the surgical act is extracted from different modalities. Each modality is registered to a 3D anatomical MRI data set. During the preoperative planning phase, the neurosurgeon selects relevant information for the surgical act from multimodal data. The selected information is transformed and stored into a file recognized by the neuronavigation system. The file contains the contours of the objects to be injected into the microscope.

Image-guided surgical procedures may be divided into four main steps: data acquisition (signals and images), data analysis (pre-planning), planning and execution of the surgical act (Figure 1). For each step we describe the actions associated with each modality and the related input and output. 


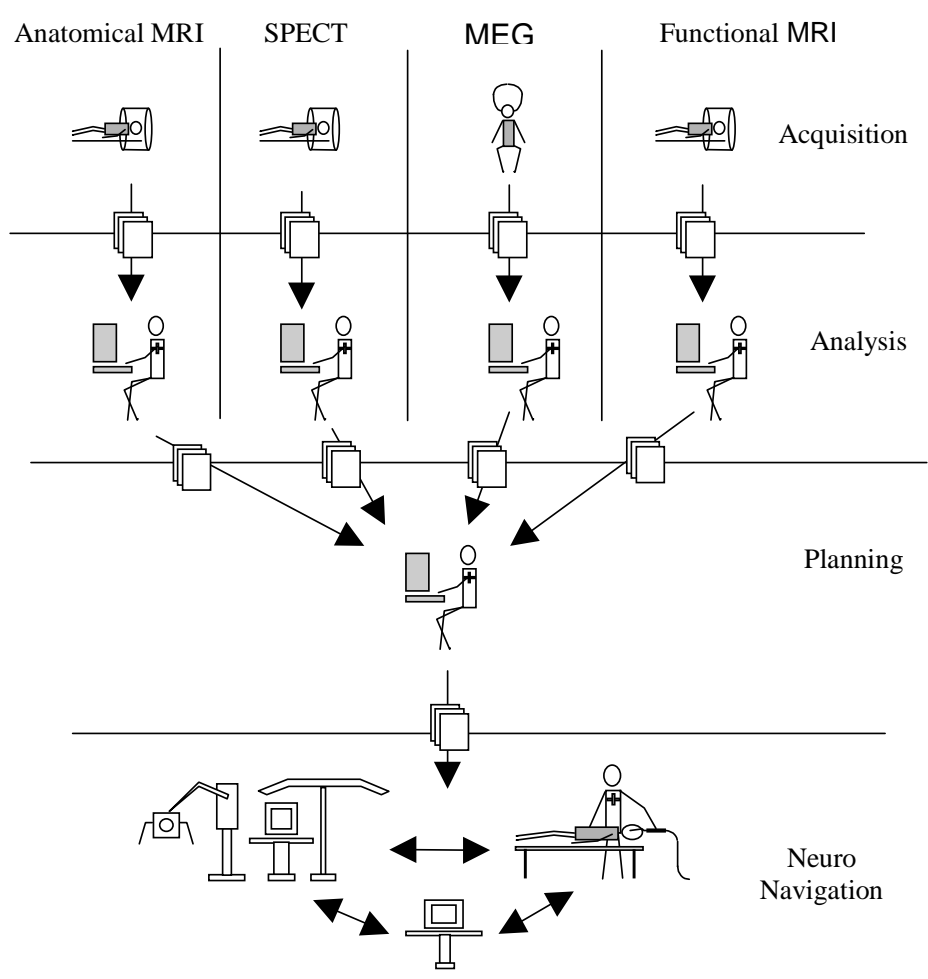

Figure 1: Data flow for multimodal neuronavigation

\subsection{Acquisition and pre-planning steps}

After acquisition comes the pre-planning step. It involves analyzing the clinical problem and selecting relevant information for the planning procedure. Since each modality is usually analyzed and segmented separately, the pre-planning step is mono-modal. In the following sections we introduce imaging protocols used for the examinations and tools we have developed to segment the entities of the surgical script.

We have developed a multimodal data base ${ }^{10}$ to store and organize image data as well as related data sets such as registration matrices, points lists (lattices) and application-related files. The data base accepts DICOM images and many other image file formats.

\subsubsection{Anatomical Imaging}

After anatomical MRI or MRA examinations, the corresponding data sets are sent to the pre-planning and planning workstation using the DICOM protocol. They are then automatically included in our database. From the anatomical MRI data set we reconstruct an isotropic volume. We have developed segmentation tools for automatic anatomical objets definition. Morphological operators are used for brain and lesional area segmentation. Classification methods are used for the segmentation of white matter, gray matter and ventricles ${ }^{8}$. We have also developed a method for the segmentation of cortical folds based on the active contours and curvature analysis ${ }^{9}$. This method allows the computation of a compact numerical description of a sulcus by modeling its median surface. Each of the extracted median surfaces is modeled by a B-spline. Vessels are segmented using morphological operators.

\subsubsection{Neuro-functional imaging}

After MEG acquisition a spatio-temporal algorithm ${ }^{2}$ is used with or without anatomical constraints for the computation of the location of equivalent current dipoles (MEG ECD). This algorithm provides either the 3D coordinates of MEG ECD or a 3D map correlation index. These results are stored in the patient data base as a list of 3D points or a 3D texture volume. fMRI data are analyzed using Functool software package (General Electric Medical Systems). A correlation volume is generated, and significant volumes of interest (VOI) with crosscorrelation values superior to 0.5 are selected by the radiologist. The images are sent to the planning workstation using the DICOM protocol and automatically included in our database. The selected VOI are sent to the planning workstation and manually included in the patients database as additional information. 


\subsection{Planning step}

The planning step includes defining the therapeutic procedure and selecting the relevant information required during the actual surgery. It involves the registration each modality to the anatomical reference MRI data set and the multimodal analysis of the previously selected information. It is a multimodal step. Due to the specifications of the SMN system, we also need to transform the volume-based or point-based representation of the entities into a surface-based representation.

\subsubsection{Registration to the anatomical reference MRI data set}

The registration step takes place in the planning workstation using the anatomical 3D SPGR MRI volume as reference volume. Every modality and information is registered to this volume. The MEG/MRI registration is computed by the STA/R software (Biomagnetic Technology Inc.). Registration uses the skin surface extracted from binarized 3D MRI and a scatter plot representing a shape acquired with a 3D magnetic localizer (Polhemus) during the MEG examination. The registration algorithm is based on Chamfer distance for cost function and on Powell's method for minimization ${ }^{17}$. A multi-resolution strategy is also used to improve registration. The coregistration of fMRI and MRI data sets is based on mutual information ${ }^{11,22}$ for cost function and on Powell's method for maximization with a multi-resolution strategy. We used a Spin-Echo anatomical sequence acquired in the same location as the functional sequence to register the fMRI images to the anatomical MRI. The MRA/MRI registration is also performed by maximizing mutual information using Powell's method for maximization with a multi-resolution strategy.

\subsubsection{Display, matching and selection of multimodal information}

In order to match different modalities, visualize 3D volumes and select entities to plan the surgical act, we have developed a set of tools included in our data fusion software environment.

\subsubsection{Matching}

This application lets you exchange 3D coordinates between pre-registered data sets. The multimodality software environment consists in a graphical user interface to load multimodal data sets and to "connect" several display modules (e.g., a 3D volume renderer, a 3D surface viewer and a digital atlas) via an application manager (router for communication between the display modules and computation of geometrical transformations). A cursor change by user interaction is immediately propagated to all the other "connected" display modules using the corresponding geometrical transformation. The transformation can be rigid or deformation field-based for either intra-patient, inter-patient or anatomical atlas fusion.

\subsubsection{3D display}

We have developed or adapted several visualization modules to fit our modular organization. A 3D ray tracer module $^{1}$ for voxel type data visualizes three-dimensional views of a volume set as well as two-dimensional sections. This module also comprises 3D texture mapping and 3D superimposition of point sets. Its functionality has been developed to provide a visual superimposition approach that lets you display several modalities on one single 3D image 7 . An Iv-viewer module based on SGI's Open-Inventor library allows 3D surface rendering. Our TalairachWin module displays digitized versions of Talairach's anatomical atlas planes ${ }^{20}$. When patient data is registered according to the Talairach's proportional squaring system, the module displays the sagittal, axial and frontal atlas planes corresponding to the 3D cursor coordinates shared by the different display modules. The label of the current point's Talairach region can also be retrieved.

\subsubsection{Selection of relevant entities}

We have developed a user interface that allows the operator to select the relevant entities for the particular surgical step among previously segmented or reconstructed entities. MEG ECDs, fMRI volumes of interest, and segmented sulci are displayed along with the MRI data set. Their specific characteristics appear in separate windows. For each modality, the user can manually select the entities required for the surgical step. He can apply filters for assistance (based on the correlation values for MEG ECD or on labels for sulci). The operator is also able to navigate, for instance, from a particular MEG ECD to its 3D location shown on the display module and from the 3D cursor to the closest corresponding fMRI VOI. Each selected entity can be transformed from its initial representation (3D points or volume) to a surface-based representation and stored in the required format for the surgical step. 


\subsubsection{Translation to surface-based representation}

The overlay of pre-operative images into the view of the surgical field through the microscope should never hamper the comprehension of the real world. The overlaid images have to be easily interpreted. These are the reasons for which preference should be given to a surface-based representation over a volume-based

representation. Therefore, information all has to be encoded into surface-based representation using appropriate methods. We have defined the best adapted 3D surface-based representation and developed the surface generation method for individual entities from every modality (Figure 2).

We represent singular MEG ECD by spheres with a pre-defined radius corresponding to a confidence area. To visualize a global functional representative area embracing a whole set of MEG ECDs, we "wrap" the dipole set

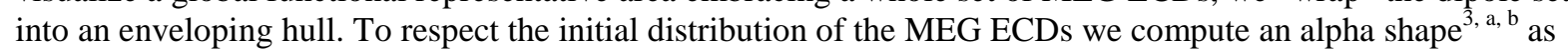
enveloping hull. For the sulci, the segmentation process directly generates a 3D surface encoded as "quadmesh". fMRI information is represented by the 3D surface of the selected VOI. Lesional areas from MRI and vessels from MRA are segmented from a 3D volume with morphological operators. We have incorporated an "Adaptive Skeleton Climbing" algorithm ${ }^{13, \mathrm{c}}$ (AMC) -a modification of the marching cubes algorithm- which creates a 3D iso-surface of these volumes.

\subsection{Surgical step}

The SMN neuronavigation system comprises a 3D digitizer and an imaging workstation. Registration of the anatomical reference MRI to the patient on the surgical site is based on non-invasive stereotactic fiducial markers attached to the patient's head. This requires the acquisition of an extra high resolution MRI data set the day before surgery. Therefore, pre-operative planning image coordinate systems and patient coordinate system are matched by composing two transformations: the transformation of a given modality into the pre-operative planning anatomical MRI and the transformation of this anatomical MRI into the anatomical MRI with markers acquired for neuronavigation. Once patient and images are registered, identified points in the patient's coordinate systems are shown on the images of the workstation (3D cursor approach). The SMN system also offers an additional visual device: a graphical overlay over the surgeon's real vision of the operating field through the microscope (visual superimposition or image overlay approach). This "augmented reality" includes the 2D mono-color display of images, graphics and symbols. The projected graphics consist in sections of selected objects, the focal plane being displayed as contours. The optics of the microscope are known with respect to the patient's position. Because the patient is registered with the preoperative images, the overlay is precisely aligned with the real world as seen through the microscope. Information sent to the system via a specific file format is displayed onto the images in the workstation as graphical 3D surfaces (Figure 5) and injected into the right ocular of the microscope as 2D green contours (Figure 4).

\section{Illustrative case reports}

We have been using the SMN system for tumoral and epilepsy surgery since 1995 . We began integrating functional information (MEG) into the neuronavigation system manually ${ }^{16}$ in 1997. Since the beginning of 1999 , we have been developing an environment allowing the automatic insertion of multimodal information into the SMN system. This environment has been used for 5 patients for epilepsy surgery or tumoral surgery when the lesional areas were close to high risk functional areas ${ }^{\mathrm{d}}$.

The first clinical case (patient A) is a 27-year-old right-handed man (Figures: 2, 3, 4, 5) who had been suffering from generalized seizures for 4 weeks. Post-ictally, his neurological functions were intact. MRI scans (1.5 T Signa General Electric Medical Systems) revealed a relatively well-circumscribed lesion $(5 * 7 \mathrm{~cm}$.). There was no enhancement in the T1-weighted 3D SPGR and T2-weighted series in the left prefrontal area. The lesion appeared to widen the medial frontal gyrus and displace the precentral sulcus posteriorly. Somatosensory, motor and language BOLD functional MRI (EPI imaging, 1.5 T Signa MR scanner, General Electric Medical Systems), along with MEG (MAGNES 37 channel Biomagnometer system, Biomagnetic Technologies Inc.) recordings were performed. Data from the different recordings for each paradigm were analyzed and the source locations were related to 3D MRI scans. The day before surgery, multimodal data was transferred to the neuronavigation system. Total tumor removal was achieved under general anesthesia. Physiopathological examination of the tissue revealed a low grade oligodendroglioma. Postoperative course was uneventful and the patient was discharged after eight days. In this case, the different steps of anatomical and functional preoperative mapping were useful to locate the sensory-motor cortex and the areas of language-related cortex. The segmented lesional

\footnotetext{
${ }^{a}$ http://www.netlib.org/voronoi/hull.html

${ }^{\mathrm{b}}$ http://cm.bell-labs.com/cm/cs/who/clarkson

${ }^{\mathrm{c}}$ http://www.cs.cuhk.edu.hk/ ttwong

${ }^{\mathrm{d}} \mathrm{http}: / /$ sim3.univ-rennes 1.fr/igs
} 
area was helpful to define and to follow the surgical path. Sulci were used to identify the anatomical environment of the surgical field. Functional information also helped determine the position of the precentral and central sulci and the position of motor and language-related areas. This determination is often difficult when normal structures are distorted by the lesion. During the surgical procedure, the use of multimodal and multiinformational neuronavigation provided helpful guidance to establish the limits of the craniotomy and to differentiate the boundary between tumor and adjacent normal brain parenchyma, especially during the removal of the deep part of the tumor.
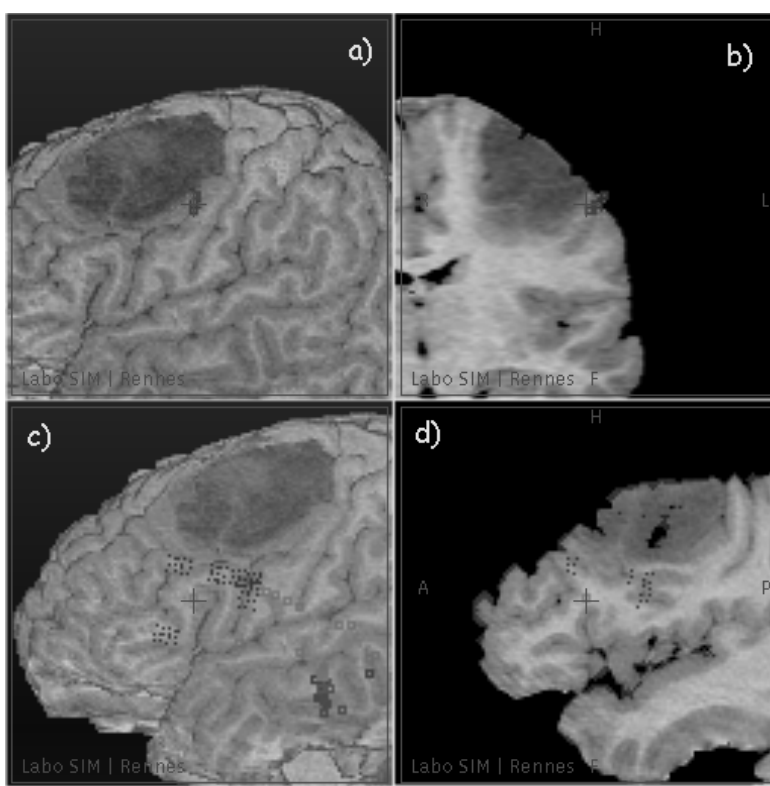

d)

Figure 2: Functional mapping of MEG and fMRI information on 3D MRI. a) and b) Motor finger tapping paradigm (fMRI: Cyan, MEG: Red and green). c) and d) Language nouns generation paradigm (fMRI: red, MEG: blue, cyan and green) (Patient A)

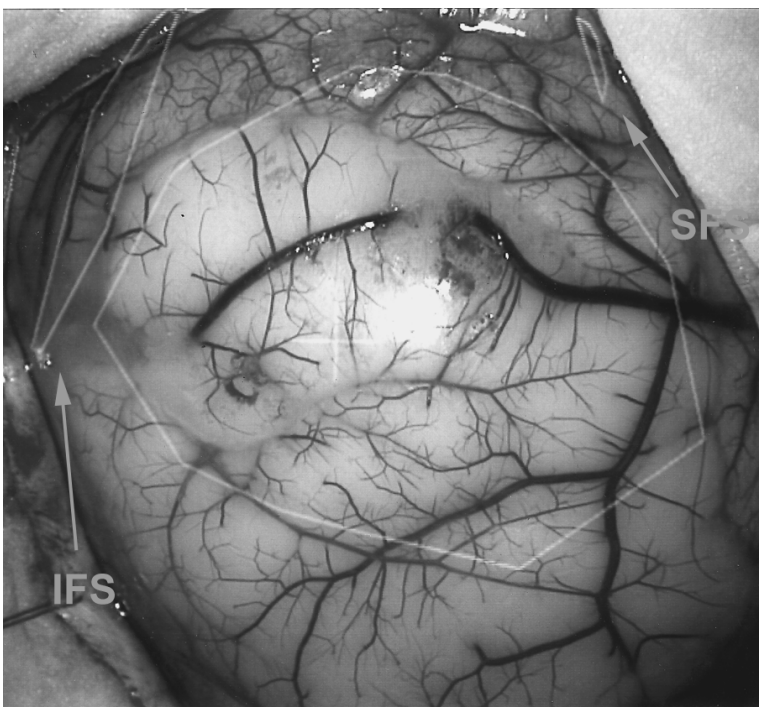

Figure 4: Intra-operative view through the microscope showed the tumor and the superior and inferior frontal sulci (Patient A)

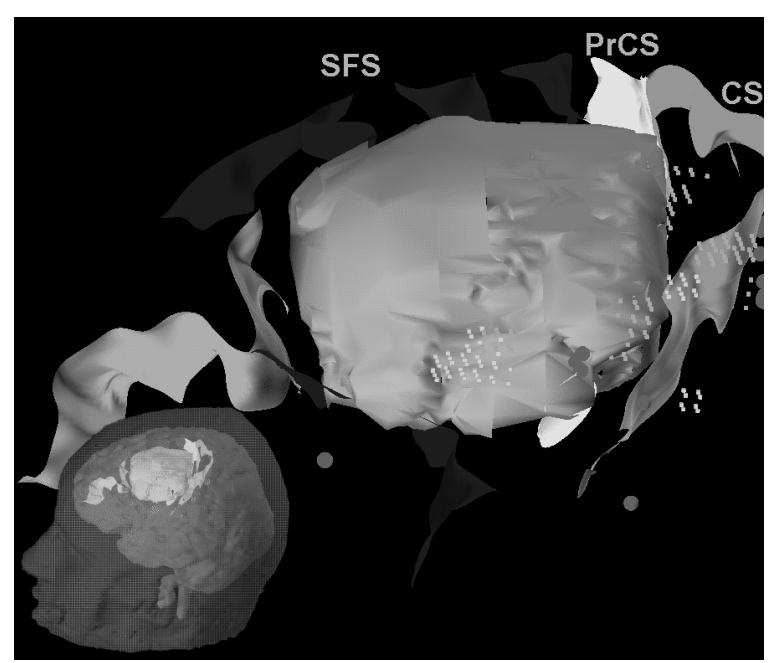

Figure 3: 3D surface-based representation of the selected entities sent to the neuronavigation system: sulci, red and pink spheres representing MEG, yellow dots representing fMRI and lesion in green (Patient A)

CS: Central Sulcus, PrCS: Pre Central Sulcus, SFS: Superior Frontal Sulcus, IFS: Inferior Frontal sulcus

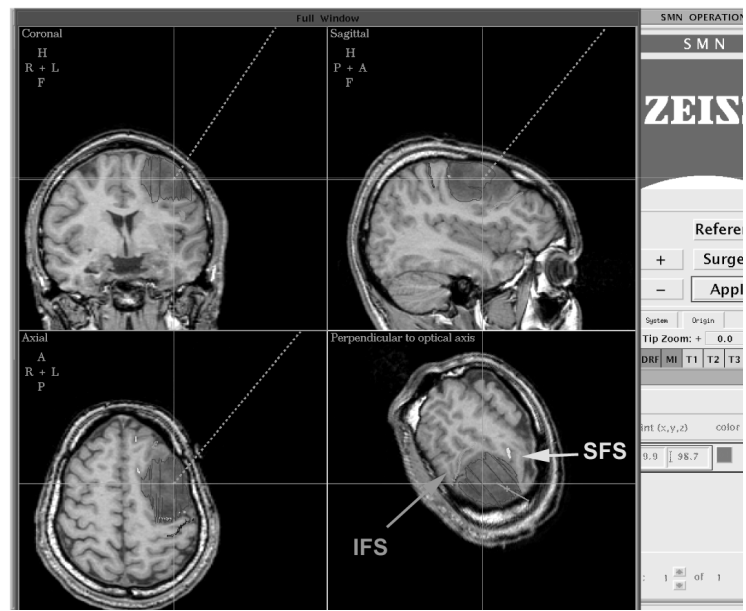

Figure 5: Intra-operative view of the STP software showing the selected entities as contours on the MRI during surgery (Patient A)

The second clinical case (patient B) is a 65 year-old right-handed woman (Figure 6) who had had a single temporal seizure with transitory aphasia. Her neurological examination did not show any neurological deficit. MRI scans revealed a left temporal meningioma ( $2 \mathrm{~cm}$ diameter). Somato-sensory, motor and language fMRI and MEG recordings were performed. Only the language information was kept and transferred to the neuronavigation system, as the results of the other paradigms were not located near the lesional area. We also selected sulci around the tumor and segmented the lateral sinus from MRA (SPGR TOF). Total tumor removal 
was achieved under general anesthesia. The physiopathological examination confirmed the diagnosis of meningioma. We did not notice any post-operative neurological deficit. In this case, the pre-operative mapping was useful to explain the post-ictal language deficit and to determine the surgery around the language-related cortex. Resection started at the part of the tumor closest to the MEG language dipoles, followed the medial temporal sulcus and ended near the veins near the lateral sinus.

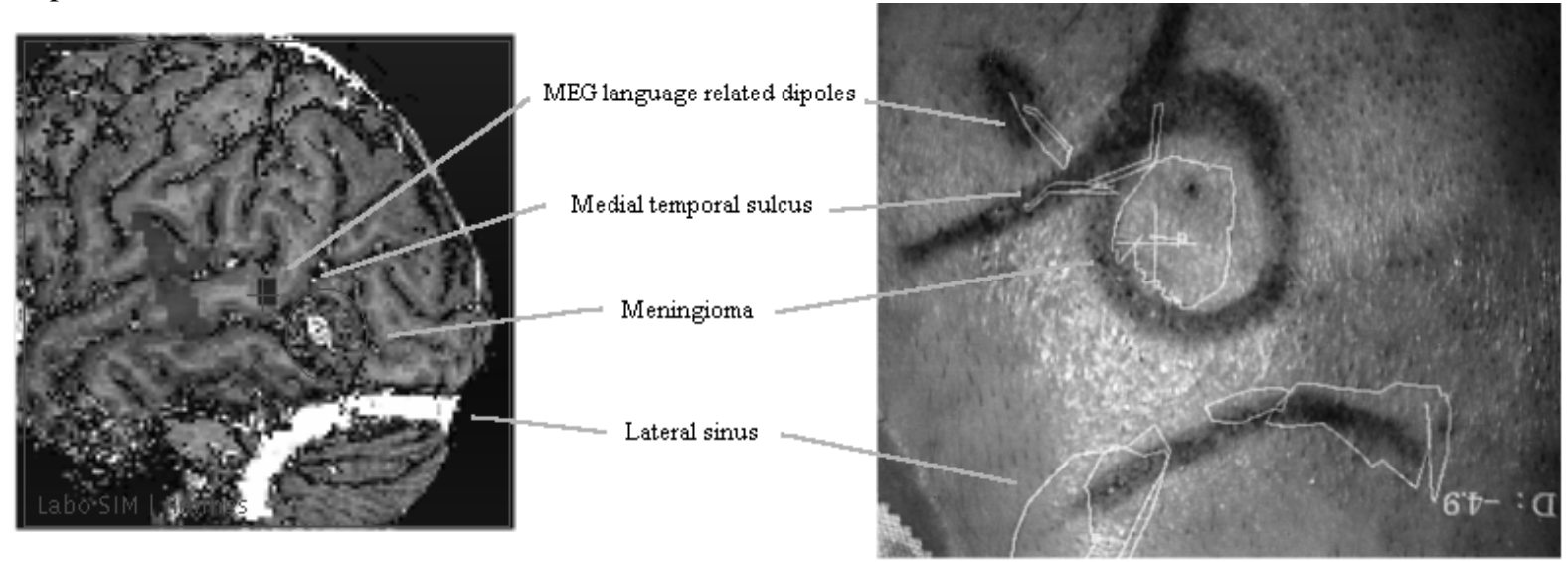

Figure 6: Pre-operative and intra-operative views of the selected entities (Patient B)

\section{Discussion}

Previously published papers on the use of functional images within neuro-navigation systems ${ }^{4,5,6,12,14,15}$ describe the manual integration of functional information into a neuronavigation system. Functional areas are drawn manually on the anatomical MRI data set during the planning step on the neuronavigation workstation. The drawings of these contours can be simplified by overwriting the MR images with functional information before exporting them to the neuronavigation workstation ${ }^{5}$.

We have developed the concept of multi-informational neuronavigation which provides the neurosurgeon with every relevant entity for the surgical procedure directly in the operating room. Information from the planning step can be displayed during the actual surgery. The information is available on the workstation as well as on the overlay in the right ocular of the microscope. We have automated the procedure from acquisition to surgery as much as possible, keeping user intervention to the minimum. Segmentation and registration are automatic and some batch computing capacities have been added. The definition of automatic procedures for segmentation and representation of multimodal information is justified by the high number of complex surgical acts requiring an important amount of multimodal information.

We have defined a generic solution that distinguishes pre-planning, planning and surgical steps. The data formats used for input and output at each step are standard (DICOM, VRML). This solution is independent from the neuronavigation system (tracking system, surgical microscope and software user interface). Our solution allows the future integration and display of new entities within the surgical environment. We are currently including Single Photon Emission Computed Tomography imaging (SPECT) for tumoral and epileptogenic area definition. Our approach also anticipates future developments of systems with superimposition of 3D and stereoscopic graphics in the microscope.

However multimodal neuronavigation remains a complex and difficult task. At each level of the procedure, it requires significant operator time to acquire the data, to analyze it and to select entities. The total amount of computation time for each registration and segmentation step is important and requires additional computing power. The generated data occupies a large amount of disk space. For instance, the first clinical case (patient A) accumulated 600 megabytes of data.

The graphical overlay is an important feature in our neuronavigation system. This augmented reality feature allows a direct confrontation between pre-operative imaging and the real view of the surgical field. The overlay is monocular and monochrome. The projected information consists in two-dimensional contours in the focal plane. There are two main limitations in this system: 1) no information about structures before or behind this plane is visible except the projection of the trajectory, the target point and its distance to the focal plane, 2) different contours can not be visualized with different colors, line widths or labels. The surgeon has to switch contours on and off to identify them. In order to take full advantage of the augmented reality, a colored stereovision overlay seems essential. Such a system ought to enhance and improve the virtual information overlaid on 
the real view without disturbing the perception of the real world. It should take into account the threedimensional nature of the pre-operative information within the microscope.

The definition or choice of a surgical path is not a trivial task and is sometimes crucial. In this study it was chosen manually with the STP software. In the future, tools that assist the surgeon in this definition need to be developed using atlas-based ${ }^{19,21}$ or simulation-based ${ }^{18}$ tools.

The reliability of the information available on our system (anatomical and functional information) is key issue. The reliability of anatomical information during the surgical act depends on the precision of registration and segmentation, while the reliability of functional information depends on 1) registration, 2) segmentation, 3) signal processing (reconstruction or localization for MEG and statistical analysis for fMRI) and 4) significance of this functional information (activation paradigms, physiological phenomenon). We are able to quantify the accuracy of the first two steps but a quantitative validation is not currently available for steps 3) and 4). The accuracy of pre-operative coregistration of a given modality and the anatomical MRI is about the lowest resolution of both modalities. According to our neuro-navigation software, the accuracy of the intra-operative registration of the patient with the images is about $1.5 \mathrm{~mm}$ (RMS), but is highly dependent on the skin markers' distribution and localization. The segmentation steps are validated by the neurosurgeon during the selection of relevant entities for the surgical performance. He can check the quality of segmentation by referring to the original 3D data sets.

Neurosurgeons are aware of the different problems with the reliability of functional information. For each clinical case both MEG and fMRI examinations were performed. The confidence associated to this functional information was higher when data was concordant. In the context of our multimodal neuronavigation system, functional information is checked with the global anatomical knowledge of the surgeon. It is only used to confirm a clinical hypothesis and to support the definition of a surgical strategy. At present a surgeon never bases his surgical approach only on functional imagery information.

\section{Conclusion}

We have designed solutions for multimodal and multi-informational neuronavigation that let surgeons access information defined during the planning step directly in the operating theatre. It is an open solution. We are convinced that in the field of multimodal neuronavigation especially, industrial products should be open to provide standard interfaces to communicate between heterogeneous hardware and software. Neuronavigation should also shift from a current technical "concept" towards a more clinical product with improved man/computer interface using a surgical dialogue. The development of 3D colored stereoscopic overlays displayed over the real view of the surgical field will improve the match between pre-operative information and the surgical environment. Today pre-operative functional imaging modalities are not completely validated. However, as functional information is available and used for pre-operative planning it has to be made accessible in the operating theatre to maximize the surgeon's knowledge of his patient.

\section{Acknowledgments}

We are grateful to Elizabeth Le Rumeur (LRBM, Faculté de Médecine, Rennes/France) for the fMRI examinations. We would also like to thank Biomagnetic Technologies Inc. (Eric Poiseau) for their technical support on magneto-encephalography for this study. This research program is supported in part by grants from the Regional Council of Brittany.

\section{References}

1. C. Barillot (1993) Surface and Volume Rendering Techniques to Display 3-D Data. IEEE Eng in Med and Biol March 1993:111-119

2. A. Bouliou, P. Bihoué, P. Toulouse, E. Poiseau, J.M. Scarabin (1995) Modelisation of the cerebral activity by a Probability Distribution upon the Cortical Surface from Spatio-Temporal MEG Data. Proc. Of Human Brain Mapping:105

3. H. Edelsbrunner, E. Mücke (1994) Three-dimensional Alpha Shapes. A.C.M. Trans. on Graphics 1:43-72

4. O. Ganslandt, R. Steinmeier, H. Kober, J. Vieth, J. Kassubek, J. Romstök, C. Strauss, R. Fahlbusch (1997) Magnetic Source Imaging Combined with Image-guided Frameless Stereotaxy: A New Method in Surgery around the Motor Strip. Neurosurgery 3:621-628

5. O. Ganslandt, R. Fahlbusch, C. Nimsky, H. Kober, M. Möller, R. Steinmeier, J. Romstök, J. Vieth (1999) Functional Neuronavigation with Magnetoencephalography: Outcome in 50 Patients with Lesions around the Motor Cortex. Journal of Neurosurgery 91:73-79 
6. M. Hardenack, N. Bucher, A. Falk, A. Harders (1998) Preoperative Planning and Intraoperative Navigation: Status Quo and Perspectives. Computer Aided Surgery 4:153-158

7. P. Jannin, C. Grova, D. Schwartz, C. Barillot, B. Gibaud (1999) Fusion of Multimodal Functional Neuroimaging (MEG, fMRI, SPECT): Definition of different Matching Paradigms. Proc. Of Computer Assisted Radiology and Surgery, H.U. Lemke and al. Eds, Springer-Verlag, 238-243

8. F. Lachmann, C. Barillot (1992) Brain Tissue Classification from MRI Data by Means of Texture Analysis. Proc. Of SPIE Medical Imaging, Vol. 1652, pp. 72-83

9. G. Le Goualher, C. Barillot, Y. Bizais (1997) Modeling Cortical Sulci with Active Ribbons. International Journal of Pattern Recognition and Artificial Intelligence 8:1295-1315

10. D. Lemoine, E. Lussot, D. Legeard, C. Barillot (1994) Multimodal Registration System for the Fusion of MRI, CT, MEG and 3D or Stereotactic Angiography Data. Proc. of SPIE Medical Imaging, 2164:46-56

11. F. Maes, A. Collignon, D. Vandermeulen, G. Marchal, P. Suetens (1997) Multimodality Image Registration by Maximization of Mutual Information. IEEE Trans. On Medical Imaging 2:187-198

12. J.A. Maldjian, M. Schulder, W. Liu, I. Mum, D. Hirschorn, R. Murthy, P. Carmel and A. Kalnin (1997) Intraoperative Functional MRI Using a Real-Time Neurosurgical Navigation System. Journal of Computer Assisted Tomography 6:910-912

13. T. Poston, T. Wong, P. Heng (1998) Multiresolution Isosurface Extraction with Adaptative Skeleton Climbing. Proc. Of Eurographics, Vol. 17, $\mathrm{N}^{\circ} 3$

14. A. Rezaï, M. Hund; E. Kronberg, M. Zonenshayn, J. Cappell, U. Ribary, B. Kall, R. Llinàs, P. Kelly (1996) The Interactive Use of Magnetoencephalography in Stereotactic Image-guided Neurosurgery. Neurosurgery 1:92-102

15. T. Roberts, E. Zusman, M. McDermott, N. Barbaro, H. Rowley (1995) Correlation of Functional Magnetic Source Imaging with Intraoperative Cortical Stimulation in Neurosurgical Patients. Journal of Image Guided Surgery 1:339-347

16. J.M. Scarabin, P. Jannin, D. Schwartz, X. Morandi (1997) MEG and 3D Navigation in Image Guided Neurosurgery. Proc. of Computer Assisted Radiology and Surgery 1997, H.U. Lemke and al. Eds, SpringerVerlag, 767-771

17. D. Schwartz, D. Lemoine, E. Poiseau and C. Barillot (1996) Registration of MEG/EEG data with 3D MRI: Methodology and Precision Issues. Brain Topography, 9/2:101-116

18. L. Serra, R. Kockro, C. Guan, N. Hern, E. Lee, C. Chan, W. Nowinski (1998) Multimodal Volume-Based Tumor Neurosurgery Planning in the Virtual Workbench. Proc. of MICCAI 98, Springer-Verlag, LNCS $\mathrm{N}^{\circ}$ 1496, W. Wells and A. Colchester Eds, pp. 1007-1015

19. P. St Jean, A. Sadikot, L. Collins, D. Clonda, R. Kasrai, A. Evans, T. Peters (1998) Automated Atlas Integration Three-Dimensional Visualization Tools for Planning and Guidance in Functional Neurosurgery. IEEE Trans. on Medical Imaging, 5:672-680

20. J. Talairach, P. Tournoux (1988) Co-Planar Stereotactic Atlas of the Human Brain. New York: Thieme,

21. M. Vaillant, C. Davatzikos, R. Taylor, R. Bryan (1997) A Path Planning Algorithm for Image-Guided Neurosurgery. Proc. of C.V.R.Med.'97, 467-476

22. W. Wells, P. Viola, H. Atsumi, R. Kikinis (1996) Multi-Modal Volume Registration by Maximisation of Mutual Information. Medical Image Analysis 1:35-51 